\title{
CYLINDRIC AND POLYADIC ALGEBRAS
}

\author{
BERNARD A. GALLER
}

1. Introduction. In recent years there have appeared two algebraizations of the first-order predicate calculus; i.e., the polyadic algebras of Halmos $[1 ; 2]$, and the cylindric algebras of Tarski $[3 ; 4]$. While polyadic algebras are the algebraic version of the pure firstorder calculus, cylindric algebras yield an algebraization of the firstorder calculus with equality. Since the pure calculus does not contain any identifiable predicate, one cannot expect to find the algebraic analogue of an equality predicate in a general polyadic algebra. It is reasonable, however, to consider "adjoining" an equality predicate, in some sense, to a polyadic algebra, and ask if one then obtains a cylindric algebra. This is the procedure followed here. An $e$-algebra is defined as a polyadic algebra with an equality predicate. We show that every $e$-algebra is in a natural way a cylindric algebra. Conversely, it is shown that in the presence of an infinite supply of variables and a local finiteness condition, cylindric algebras are in a natural way $e$-algebras, and the correspondence obtained in this way between $e$-algebras and cylindric algebras is one-to-one.

2. Polyadic algebras. A quantifier (or, more explicitly, an existential quantifier) on a Boolean algebra $A$ is a mapping $\exists: A \rightarrow A$ such that (1) $\exists 0=0$, (2) $p \leqq \exists p$, and (3) $\exists(p \wedge \exists q)=\exists p \wedge \exists q$ for all $p, q \in A$. A polyadic algebra is a quadruple $(A, I, S, \exists)$, where $A$ is a Boolean algebra, $I$ an arbitrary set whose elements are called variables, $S$ is a mapping from transformations of $I$ into itself to Boolean endomorphisms on $A$ (the transformations need not be one-to-one nor onto), and $\exists$ is a mapping from subsets of $I$ to quantifiers on $A$, satisfying the following conditions:

$\left(\mathrm{P}_{1}\right) \exists(\varnothing) p=p$ for all $p \in A$ ( $\varnothing$ shall denote the empty set throughout).

$\left(\mathrm{P}_{2}\right) \exists(J \cup K)=\exists(J) \exists(K)$ for all subsets $J$ and $K$ of $I$.

$\left(\mathrm{P}_{3}\right) S(\delta)=f$ (where $\delta$ is the identity transformation on $I$ and $f$ is the identity endomorphism on $A$ ).

$\left(\mathrm{P}_{1}\right) S(\sigma) S(\tau)=S(\sigma \tau)$ for all transformations $\sigma$ and $\tau$ on $I$.

$\left(\mathrm{P}_{5}\right)$ If $J \subset I$ and $\sigma$ and $\tau$ are transformations on $I$ which agree outside $J$, then $S(\sigma) \exists(J)=S(\tau) \exists(J)$.

$\left(\mathrm{P}_{6}\right)$ If $J \subset I$ and $\tau$ is a transformation which is one-to-one on $\tau^{-1} J$, then $\exists(J) S(\tau)=S(\tau) \exists\left(\tau^{-1} J\right)$.

Presented to the Society, April 22, 1955; received by the editors July 22, 1955 and, in revised form, March 28, 1956. 
If $p \in A$, then $p$ will be said to be supported by the set $J$ if $\exists(I-J) p$ $=p$. We will say that $p$ is independent of the set $K$ if $\exists(K) p=p$, so that $J$ supports $p$ if and only if $p$ is independent of $I-J$. A polyadic algebra will be called locally finite if each element $p$ of the algebra is supported by some finite set $J_{p}$. A transformation $\tau$ will be called finite if $\tau$ agrees with $\delta$ outside some finite set. If $i$ and $j$ are elements of $I$, the transformation which maps $i$ onto $j$ and every other element of $I$ (including $j$ ) onto itself will be called a replacement and denoted by $(i / j)$. If $I$ is infinite, the algebra will be said to have infinite degree. A quasi-polyadic algebra is a quadruple $(A, I, S, \exists)$, where $A$ is a Boolean algebra, $I$ a set, $S$ a mapping from finite transformations on $I$ to Boolean endomorphisms on $A$, and $\exists$ is a mapping from finite subsets of $I$ to quantifiers on $A$, satisfying the conditions:

$\left(Q_{1}\right) \exists(\varnothing) p=p$ whenever $p \in A$. I.

$\left(Q_{2}\right) \exists(J \cup K)=\exists(J) \exists(K)$ whenever $J$ and $K$ are finite subsets of

$\left(\mathrm{Q}_{3}\right) S(\delta)=f$.

$\left(Q_{4}\right) S(\sigma) S(\tau)=S(\sigma \tau)$ whenever $\sigma$ and $\tau$ are finite transformations on $I$.

$\left(Q_{5}\right)$ If $\sigma$ and $\tau$ are finite transformations on $I$, if $J$ is a finite subset of $I$, and $\sigma=\tau$ outside $J$, then $S(\sigma) \exists(J)=S(\tau) \exists(J)$.

$\left(Q_{6}\right)$ If $\tau$ is a finite transformation on $I$, if $J$ is a finite subset of $I$, and if $\tau$ is one-to-one on $\tau^{-1} J$, then $\exists(J) S(\tau)=S(\tau) \exists\left(\tau^{-1} J\right)$.

$\left(Q_{7}\right)$ If $p \in A$, then there exists a cofinite set $J$ (i.e., $I-J$ is a finite set) such that $\exists(K) p=p$ whenever $K$ is a finite subset of $J$.

We shall need the following result concerning quasi-polyadic algebras from [2].

Theorem. If $(A, I, S, \exists)$ is a quasi-polyadic algebra, then (i) there exists a mapping $S^{*}$ from transformations on $I$ to Boolean endomorphisms of $A$ such that $S^{*}(\tau)=S(\tau)$ whenever $\tau$ is a finite transformation, (ii) there exists a mapping $\Xi^{*}$ from subsets of $I$ to quantifiers on $A$ such that $\exists^{*}(J)=\exists(J)$ whenever $J$ is a finite set, (iii) the quadruple $\left(A, I, S^{*}, \exists^{*}\right)$ is a locally finite polyadic algebra, and (iv) the mappings $S^{*}$ and $\Xi^{*}$ are uniquely determined by (i), (ii), and (iii).

We shall also need the fact, established in [2], that if $\tau$ is a finite transformation on $I$ and $J$ is a finite subset of $I$, then there is a finite ordered collection $\left\{\tau_{1}, \cdots, \tau_{n}\right\}$ of replacements on $I$ such that $\tau=\tau_{1} \cdots \tau_{n}$ on $J$.

3. e-Algebras. If $e($,$) is the equality predicate for the first-order$ functional calculus with equality, then it is well known that $e($,$) is$ characterized by the reflexive and substitution properties. Moreover, 
if we have $e(x, y)$, then the transformation which maps $x$ onto $z$ yields the equality of $z$ and $y$; i.e., $e(z, y)$. More generally, the effect on $e(x, y)$ of a transformation on the variables is obtained by allowing the transformation to act on the variables $x$ and $y$ directly. These considerations furnish the motivation for Definitions 1 and 2. (Condition (2) of Definition 2 asserts essentially that if $p$ is true and $i=j$, then $p$ is true with $i$ replaced by $j$; i.e., the substitution property.)

Definition 1. Let $(A, I, S, \exists)$ be a polyadic algebra. A binary predicate for $A$ is a function $p: I \times I \rightarrow A$ such that $S(\tau) p(i, j)=p(\tau i, \tau j)$ for every transformation $\tau$ on $I$.

Definition 2. A polyadic algebra with equality (or, an e-algebra) is a polyadic algebra $(A, I, S, \exists)$ for which there exists a binary predicate $e$ for $A$ such that (1) $e(i, i)=1$ for all $i \in I$, and (2) $p \wedge e(i, j)$ $\leqq S(i / j) p$ for all $i, j \in I$ and $p \in A$. We shall denote the $e$-algebra by $(A, I, S, \exists, e)$.

Definition 3. A cylindric algebra is a Boolean algebra $A$, together with a function $C$ from a set $I$ to quantifiers on $A$, and a function $d: I \times I \rightarrow A$ such that (1) $C(h) C(j)=C(j) C(h),(2) d(i, i)=1,(3) d(i, j)$ $=C(k)[d(i, k) \wedge d(j, k)]$, and (4) $C(i)[p \wedge d(i, k)] \wedge C(i)\left[p^{\prime} \wedge d(i, k)\right]$ $=0$ whenever $i, j, h, k$ are elements of $I$ such that $i \neq k$ and $j \neq k$. The cylindric algebra will be denoted by $(A, I, C, d)$.

Definition 4. A cylindric algebra $(A, I, C, d)$ will be called locally finite if for each $p \in A$, the set $\{j \in I \mid C(j) p=p\}$ is cofinite.

We note that Definitions 3 and 4 are in an obvious way equivalent to the definitions given by Tarski in [3].

Definition 5. An $e$-algebra $(A, I, S, \exists, e)$ will be called cylindrizable if there exists a cylindric algebra $\left(A_{1}, I_{1}, C, d\right)$ such that $A_{1}=A, I_{1}=I, d=e$, and $C(i)=\exists(i)$ for all $i \in I$.

Definition 6. A cylindric algebra $(A, I, C, d)$ will be called equalizable if there exists an $e$-algebra $\left(A_{1}, I_{1}, S, \exists, e\right)$ such that $A_{1}=A, I_{1}=I, e=d, S(i / j) p=C(i)[p \wedge d(i, j)]$ whenever $i \neq j$, and $\exists(i)=C(i)$ for all $i \in I$.

Let $(A, I, S, \exists, e)$ be an $e$-algebra. We shall need the following lemmas.

Lemma 1. Whenever $i \neq j, S(i / j) p=\exists(i)[p \wedge e(i, j)]$ for all $p \in A$.

Proof. $\exists(i)[p \wedge e(i, j)] \leqq \exists(i) S(i / j) p=S(i / j) p$, since $i \neq j$. Also, $S(i / j) p=S(i / j) p \wedge e(j, j)=S(i / j)[p \wedge e(i, j)] \leqq S(i / j) \exists(i)[p \wedge e(i, j)]$ $=\exists(i)[p \wedge e(i, j)]$.

Lemma 2. For all $i, j \in I, e(i, j)=e(j, i)$. 
Proof. By symmetry, it is sufficient to show that $e(i, j) \leqq e(j, i)$ for all $i, j \in I$. But $e^{\prime}(j, i) \wedge e(i, j) \leqq S(i / j) e^{\prime}(j, i)=e^{\prime}(j, j)=0$.

TheOREM 1. Every e-algebra is cylindrizable.

Proof. Let $(A, I, S, \exists, e)$ be an $e$-algebra. We define $d=e$, and let $C(k)=\exists(k)$ for all $k \in I$. It is clear that $C$ maps $I$ into quantifiers on $A$ which commute, and that $d(i, i)=e(i, i)=1$ for all $i \in I$. If $i \neq k$, $j \neq k$, then, by Lemmas 1 and $2, C(k)[d(i, k) \wedge d(j, k)]=\exists(k)[e(i, k)$ $\wedge e(k, j)]=S(k / j) e(i, k)=e(i, j)=d(i, j)$. Finally, if $i \neq k$ and $p \in A$, we have $\exists(i)[p \wedge d(i, k)] \wedge \exists(i)\left[p^{\prime} \wedge d(i, k)\right]=S(i / k) p \wedge S(i / k) p^{\prime}$ $=S(i / k)\left(p \wedge p^{\prime}\right)=0$, so that $(A, I, C, d)$ is a cylindric algebra.

4. Cylindric algebras. Let $(A, I, C, d)$ be a locally finite cylindric algebra with $I$ infinite. We shall show that $(A, I, C, d)$ is equalizable. We let $e=d, \quad \exists(\varnothing) p=p, \quad \exists(j)=C(j), \quad S(j / j) p=p$, and $S(i / k) p$ $=C(i)[p \wedge d(i, k)]$ whenever $p \in A$, and $i, j, k \in I$ such that $i \neq k$, and we define $S(\tau) p$ for $p \in A$ and $\tau$ a finite transformation on $I$, by finding a finite set of replacements on $I$, say $\left\{\tau_{1}, \cdots, \tau_{n}\right\}$ such that $\tau=\tau_{1} \cdots \tau_{n}$ on some finite support of $p$ and letting $S(\tau) p=S\left(\tau_{1}\right) \cdots$ $S\left(\tau_{n}\right) p$. Such a finite set of replacements exists, as we have remarked above, but it will be necessary to show that the definition is unambiguous. If $J=\left\{j_{1}, \cdots, j_{n}\right\}$ is a finite subset of $I$, we define $\exists(J)$ by the equation $\exists(J)=C\left(j_{1}\right) \cdots C\left(j_{n}\right)$. Since the values of $C$ commute, and since (as is easily verified) the product of two commuting quantifiers is again a quantifier, $\exists(J)$ is unambiguously defined and is a quantifier.

The proofs of the next four lemmas consist of straightforward computations, and are omitted.

Lemma 3. If $i \neq j$, then $S(i / j)$ is a Boolean endomorphism on $A$.

Leмma 4. (1) Whenever $i \neq j, \quad k \neq j, \quad S(i / k) \exists(j)=\exists(j) S(i / k)$, (2) $S(j / i) \exists(j)=\exists(j)$ for all $i, j \in I$, and (3) $\exists(j) S(j / i)=S(j / i)$ whenever $i \neq j$.

Leмma 5. If $i, j, k, h$ are distinct elements of $I$, then (1) $S(i / j) S(k / h)$ $=S(k / h) S(i / j)$, (2) $S(k / h) S(k / j)=S(k / j)$, (3) $S(k / j) S(k / j)=S(k / j)$, (4) $S(i / j) S(k / i)=S(k / j) S(i / j),(5) S(i / j) S(k / j)=S(k / j) S(i / j)$.

Lemma 6. If $\exists(j) p=p$, then $S(j / i) S(i / j) p=p$ whenever $p \in A$ and $i, j \in I$.

Definition 7. Let $\alpha$ be an ordered collection consisting of an even number of replacements on $I$, say $\alpha=\left\{\alpha_{1}, \cdots, \alpha_{2 n}\right\}, n \geqq 0$, and $J$ a finite subset of $I$. We shall say that $\alpha$ is $J$-normal if there are distinct 
elements $k_{1}, \cdots, k_{n} \in J$, distinct elements $i_{1}, \cdots, i_{n} \in I-J$, and (not necessarily distinct) elements $j_{1}, \cdots, j_{n} \in J$ such that (1) $\alpha_{r}$ $=\left(i_{r} / j_{r}\right)$, and $(2) \alpha_{n+r}=\left(k_{r} / i_{r}\right), r=1,2, \cdots, n$.

Definition 8. If $\alpha=\left\{\alpha_{1}, \cdots, \alpha_{n}\right\}$ and $\beta=\left\{\beta_{1}, \cdots, \beta_{m}\right\}$ are finite ordered collections of replacements on $I, J$ any subset of $I$, and $p \in A$, we will say that $\alpha$ and $\beta$ are $(p, J)$-equivalent if (1) $\alpha_{1} \cdots \alpha_{n} j$ $=\beta_{1} \cdots \beta_{m} j$ whenever $j \in J$, and (2) $S(\alpha) p=S(\beta) p$, where $S(\alpha)$ $=S\left(\alpha_{1}\right) \cdots S\left(\alpha_{n}\right)$ and $S(\beta)=S\left(\beta_{1}\right) \cdots S\left(\beta_{m}\right)$. If $\alpha$ and $\beta$ are $(p, I)$ equivalent for every $p \in A$, we shall say that $\alpha$ and $\beta$ are equivalent.

Definition 9. If $(i / j)$ is a replacement on $I$, we shall refer to $i$ as the essential domain of $(i / j)$, and to $j$ as the essential range of $(i / j)$.

Lemma 7 enables one to study the effect of a finite transformation $\tau$ on a finite set by examining the image of each element separately. The method is one commonly used in mathematical logic; i.e., mapping the element $i$ first into another element $j$ far from the scene of the action, and then mapping $j$ into $\tau(i)$.

Lemma 7. Let $\alpha$ be a finite ordered collection of replacements, $p \in A$, and $J$ a finite support of $p$ which contains all essential domains and essential ranges of elements of $\alpha$. Then there exists a finite ordered collection $\phi$ of replacements which is $J$-normal and $(p, J)$-equivalent to $\alpha$. Moreover, if $\phi=\left\{\phi_{1}, \cdots, \phi_{2 m}\right\}$, the essential domains of $\phi_{1}, \cdots, \phi_{m}$ may be chosen arbitrarily from $I-J$, provided they are distinct, and the essential domains of $\phi_{m+1}, \cdots, \phi_{2 m}$ are all elements of $J$.

Proof. The proof consists of successively transforming $\alpha$ into various ordered collections, the last of which is $\phi$, with the property that each is $(p, J)$-equivalent to the preceding one. The details are omitted.

Lemma 8 states essentially that if two transformations agree on a (finite) set $P$ which supports an element $q$ of $A$, except possibly on a subset $K$ of $P$ of which $q$ is independent, then they produce the same effect on $q$.

Lemma 8. Let $\alpha=\left\{\alpha_{1}, \cdots, \alpha_{n_{1}}\right\}$ and $\alpha^{*}=\left\{\alpha_{1}^{*}, \cdots, \alpha_{m_{1}}^{*}\right\}$ be finite ordered collections of replacements on $I, p \in A, P$ a finite support of $p$, and $K$ any finite subset of $I$, such that $\alpha_{1} \cdots \alpha_{n_{1}} j=\alpha_{1}^{*} \cdots \alpha_{m_{1}}^{*} j$ whenever $j \in P-K$. Then $S(\alpha) \exists(K) p=S\left(\alpha^{*}\right) \exists(K) p$ (cf. Definition 8).

Proof. Applying Lemma 7 to $\alpha$ and $\alpha^{*}$ and a finite set $J$ which contains $P$ and satisfies the hypotheses of Lemma 7 with respect to $\alpha$ and $\alpha^{*}$, we obtain finite ordered collections $\beta$ and $\beta^{*}$. These in turn can be transformed into collections $\gamma$ and $\gamma^{*}$ such that $\gamma=\gamma^{*}$. Since $\alpha, \beta, \gamma$ and $\alpha^{*}, \beta^{*}, \gamma^{*}$ are seen to be ( $\left.\exists(K) p, J\right)$-equivalent, it 
follows that $S(\alpha) \exists(K) p=S(\beta) \exists(K) p=S(\gamma) \exists(K) p=S\left(\gamma^{*}\right) \exists(K) p$ $=S\left(\beta^{*}\right) \exists(K) p=S\left(\alpha^{*}\right) \exists(K) p$.

Corollary 1. The definition of $S(\tau)$ is unambiguous for every finite transformation $\tau$.

Proof. Let $\alpha=\left\{\alpha_{1}, \cdots, \alpha_{n}\right\}$ and $\alpha^{*}=\left\{\alpha_{1}^{*}, \cdots, \alpha_{m}\right\}$ be finite ordered collections of replacements such that $\alpha_{1} \cdots \alpha_{n}=\tau$ on the finite support $Q$ of $p$, and $\alpha_{1}^{*} \cdots \alpha_{m}^{*}=\tau$ on the finite support $Q^{*}$ of $p$. Then $P=Q \cap Q^{*}$ is a finite support of $p$, and $\alpha_{1} \cdots \alpha_{n}=\tau=\alpha_{1}^{*} \cdots \alpha_{m}^{*}$ on $P$. We apply Lemma 8 with $K=\varnothing$, to obtain $S(\alpha) p=S\left(\alpha^{*}\right) p$.

COROLlaRy 2. If $\sigma, \tau$ are finite transformations on I which agree outside a finite set $K$, then $S(\sigma) \exists(K)=S(\tau) \exists(K)$.

Proof. Let $p \in A$. We find a finite ordered collection $\alpha$ $=\left\{\alpha_{1}, \cdots, \alpha_{n}\right\}$ of replacements such that $\alpha_{1} \cdots \alpha_{n}=\sigma$ on a finite support $P_{1}$ of $p$, and a finite ordered collection $\beta=\left\{\beta_{1}, \cdots, \beta_{m}\right\}$ such that $\beta_{1} \cdots \beta_{m}=\tau$ on a finite support $P_{2}$ of $p$. Then $\alpha_{1} \cdots \alpha_{n}$ $=\beta_{1} \cdots \beta_{m}$ on $P-K$, where $P=P_{1} \cap P_{2}$. It follows from Lemma 8 that $S(\sigma) \exists(K) p=S(\alpha) \exists(K) p=S(\beta) \exists(K) p=S(\tau) \exists(K) p$.

LemMa 9. Let $\tau$ be a finite transformation on $I, J$ a finite subset of $I$. If $\tau$ is one-to-one on $\tau^{-1} J$, then $S(\tau) \exists\left(\tau^{-1} J\right)=\exists(J) S(\tau)$.

Proof. If $J=\varnothing$, the lemma is trivial. Assume first that $J=\{j\}$. Let $p \in A$, and let $\alpha$ be a finite ordered collection of replacements, $\alpha=\left\{\alpha_{1}, \cdots, \alpha_{n}\right\}$, such that (1) $\alpha_{1} \cdots \alpha_{n}=\tau$ on a support $K_{1}$ of $p$, and (2) $\left(\alpha_{1} \cdots \alpha_{n}\right)^{-1} j=\tau^{-1} j$. (It is possible to find such a collection, for example, by considering $\tau \mid(I-J)$.) Let $K$ be a finite support of $p$ which includes all essential domains and essential ranges of elements of $\alpha$, as well as $j$ and $k=\tau^{-1} j=\left(\alpha_{1} \cdots \alpha_{n}\right)^{-1} j$. By Lemma 7, we can find a finite ordered collection of replacements $\beta=\left\{\beta_{1}, \cdots, \beta_{2 m}\right\}$ which is $K$-normal and $(\exists(j) p, K)$-equivalent to $\alpha$. The proof for $J=\{j\}$ then follows from Lemmas 4,5 , and 6 by consideration of the cases $j=k$ and $j \neq k$.

If $J=\left\{j_{1}, \cdots, j_{n}\right\}, n \geqq 1$, and the lemma holds for all sets $J_{1}$ with fewer than $n$ elements, let $\tau$ be one-to-one on $\tau^{-1} J$. Then $\tau$ is one-toone on $\tau^{-1} j_{1}$ and on $\tau^{-1} J_{1}$, where $J_{1}=J-\left\{j_{1}\right\}$, and $\exists(J) S(\tau)$ $=\exists\left(J_{1}\right) \exists\left(j_{1}\right) S(\tau)=\exists\left(J_{1}\right) S(\tau) \exists\left(\tau^{-1} j_{1}\right)=S(\tau) \exists\left(\tau^{-1} J_{1} \cup \tau^{-1} j_{1}\right)=S(\tau)$ $\exists\left(\tau^{-1} J\right)$.

We are now in a position to prove the principal theorem.

THEOREM 2. Every locally finite cylindrical algebra of infinite degree is equalizable. 
Proof. Let $(A, I, C, d)$ be a locally finite cylindric algebra with $I$ infinite. We define $e, S$, and $\exists(j)$ for $j \in I$ as in Definition 6 , and for $J$ finite, $J=\left\{j_{1}, \cdots, j_{n}\right\}$, we define $\exists(J)=C\left(j_{1}\right) \cdots C\left(j_{n}\right)$. It follows from our earlier remarks and Corollary 1 to Lemma 8 that the definitions of $\exists(J)$ and $S(\tau)$ are unambiguous and that $\exists(J)$ is a quantifier for any finite subset $J$ of $I$ and any finite transformation $\tau$ on $I$. An easy induction based on Lemma 3 shows that $S(\tau)$ is a Boolean endomorphism for any finite $\tau$. We shall see that the postulates for a quasi-polyadic algebra are satisfied by $(A, I, S, \exists)$, and it will follow from the theorem on quasi-polyadic algebras quoted above that $(A, I, S, \exists)$ determines a unique polyadic algebra.

Since $S(j / j) p=p$ for all $j \in I$ and $p \in A$, it follows that $S(\delta) p=p$, so that $Q_{1}$ holds. Postulates $Q_{2}, Q_{3}, Q_{4}$, and $Q_{7}$ follow immediately from the definitions, and $Q_{6}$ is Corollary 2 to Lemma 8 , while $Q_{6}$ is Lemma 9. We must show that $e$ is a binary predicate satisfying conditions (1) and (2) of Definition 2. Since $e=d$, we know that $e$ maps $I \times I$ into $A$, and $e(i, i)=1$ for all $i \in I$. To show that $S(\tau) e(i, j)=e(\tau i, \tau j)$, it follows from the definition of $S$ that it is sufficient to verify the equation $S(k / h) e(i, j)=e((k / h) i,(k / h) j)$ for all $i, j, h, k \in I$. If $k \notin\{i, j\}$ or if $k=h$, then the equation holds trivially, since $e(i, j)$ is supported by $\{i, j\}$. Suppose, then, that $k=i, k \neq h$. Then $S(k / h) e(i, j)$ $=\exists(i)[d(i, j) \wedge d(i, h)]=\exists(i)[d(j, i) \wedge d(h, i)]=d(j, h)=d(h, j)$ $=e((k / h) i,(k / h) j)$. The case $k=j, k \neq h$ is similar. Now suppose $p \in A$. Then $p \wedge e(i, j) \leqq \exists(i)[p \wedge d(i, j)]=S(i / j) p$ whenever $i \neq j$, and the inequality holds trivially when $i=j$.

Theorem 3. Let $\mathfrak{A}=(A, I, S, \exists, e)$ be a locally finite e-algebra of infinite degree. Let $\mathfrak{B}=(A, I, C, d)$ be the locally finite cylindric algebra of infinite degree arising from $\mathfrak{A}$ by means of Definition 5 ( $c f$. Theorem 1). Let $\mathfrak{A}_{1}$ be the e-algebra arising from $\mathfrak{B}$ by means of Definition 6 (cf. Theorem 2). Then $\mathfrak{A}_{1}=\mathfrak{A}$.

Proof. Let $\mathfrak{A}_{1}=\left(A, I, \exists_{1}, S_{1}, e_{1}\right)$. It follows from definitions that $\exists_{1}(k)=C(k)=\exists(k)$ for all $k \in I$, and therefore that $\exists_{1}(J)=\exists(J)$ for any finite $J$. Also, we have $e_{1}=d=e$. If $p \in A$ and $i, j \in I, i \neq j$, then $S_{1}(i / j) p=C(i)[p \wedge d(i, j)]=\exists(i)[p \wedge e(i, j)]=S(i / j) p$ by the definition of $S_{1}$ and Lemma 1 . An easy induction shows that $S_{1}(\tau)=S(\tau)$ for any finite $\tau$. The theorem follows from the uniqueness assertion of the theorem on quasi-polyadic algebras.

TheOREM 4. Let $\mathfrak{B}=(A, I, C, d)$ be a locally finite cylindric algebra of infinite degree. Let $\mathfrak{A}=(A, I, S, \exists, e)$ be the e-algebra arising from $\mathfrak{B}$ by means of Definition 6 ( $c f$. Theorem 2). Let $\mathfrak{B}_{1}=\left(A, I, C_{1}, d_{1}\right)$ be the 
cylindric algebra arising from $\mathfrak{A}$ by means of Definition 5 (cf. Theorem 1). Then $\mathfrak{B}=\mathfrak{B}_{1}$.

Proof. From the definitions, we have $d_{1}=e=d$, and $C_{1}(k)=\exists(k)$ $=C(k)$ for all $k \in I$.

\section{BIBLIOGRAPHY}

1. P. R. Halmos, Polyadic Boolean algebras, Proc. Nat. Acad. Sci. U.S.A. vol. 40 (1954) pp. 296-301.

2. - Algebraic logic, II, to appear in Fund. Math. in 1957.

3. A. Tarski and F. B. Thompson, Some general properties of cylindric algebras, Bull. Amer. Math. Soc. Abstract 58-1-85.

4. A. Tarski, $A$ representation theorem for cylindric algebras, Bull. Amer. Math. Soc. Abstract 58-1-86.

University of Chicago 